\title{
Técnica manos libres para colocación de tornillos transpediculares en columna torácica, revisión narrativa de la literatura
}

\author{
Free hand technique for placement of transpedicular screws in \\ thoracic spine, narrative review of the literature
}

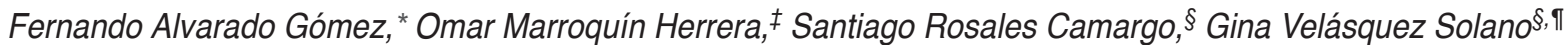
${ }^{*}$ Cirujano de Columna, Director Fellowship AOSpine, Jefe de Departamento sección de Columna, Departamento de Ortopedia y Traumatología. ORCID: https://orcid.org/0000-0002-8854-0356; ¥Fellow Cirugía de Columna AOSpine. ORCID: https://orcid.org/0000-0003-4159-0222; \$Médico Rural de Columna. ORCID: https://orcid.org/0000-0002-4591-0134; "Médico Rural de Columna. ORCID: https://orcid.org/0000-0002-8673-9062. Hospital Universitario de la Fundación Santa Fe de Bogotá, Colombia.

\begin{abstract}
Resumen
Artículo que presenta una revisión estructurada de la literatura médica donde se explica la técnica de manos libres para la colocación de tornillos transpediculares, conjunto de recomendaciones, principales complicaciones, aplicabilidad en diversas patologías y curvas de aprendizaje reportadas en dicha documentación.
\end{abstract}

Palabras clave: Tornillos transpediculares, técnica de manos libres, columna torácica.

Abstract

Structured literature review article explaining the hands-free technique for transpedicular screw placement, set of recommendations, main complications, applicability in various pathologies and learning curves reported in said documentation.

Keywords: Transpedicular screws, free-hand technique, thoracic spine.

\section{Introducción}

El uso de los tornillos transpediculares empezó con Boucher, quien utilizaba este tipo de material en la región lumbar. ${ }^{1}$ Con el paso del tiempo, tales sujetadores transpediculares se modificaron para la columna torácica. Al resaltar la importancia de las tres columnas descritas por Denis, ${ }^{2}$ el sistema pedicular torácico marca la pauta en la corrección de deformidades y patologías degenerativas, por lo cual se inició el uso de tornillería transpedicular con diversas técnicas, como el uso de radiografía convencional transquirúrgica y la fluoroscopia transquirúrgica. Estos métodos implican una mayor exposición a la radiación, mayor tiempo quirúrgico y gasto económico. La metodología de manos libres exige experticia y conocimiento de la anatomía vertebral, con la que puede disminuir el tiempo quirúrgico, la exposición a radiaciones y con ello mayor seguridad para el equipo operatorio. ${ }^{3}$

La técnica de manos libres para la colocación de sujetadores transpediculares es un procedimiento que requiere un gran conocimiento y curva de aprendizaje, dada la compleja anatomía de la región torácica vertebral. Durante esta revisión de la literatura, se explicará el sistema de instalación

Correspondencia:

Dr. Omar Marroquín Herrera

E-mail: dr.omarmhspine@gmail.com

Recibido: 23-04-2021. Aceptado: 12-05-2021.

Citar como: Alvarado GF, Marroquín HO, Rosales CS, Velásquez SG. Técnica manos libres para colocación de tornillos transpediculares en columna torácica, revisión narrativa de la literatura. Orthotips. 2021; 17 (3): 155-161. https://dx.doi.org/10.35366/100626 
de tornillos transpediculares, su clasificación de posicionamiento, la aplicabilidad que tienen en diferentes tipos de patología, la curva de aprendizaje que debe tomar el cirujano de columna al usar esta técnica, las complicaciones que pueden presentarse y, por último, se recomienda que los cirujanos de columna recién formados utilicen herramientas de ayuda y otro tipo de alternativas que le generen confianza al momento de abordar la modalidad de manos libres, con el fin de evitar complicaciones.

\section{Material y métodos}

Se realizó una búsqueda estructurada de la literatura médica pertinente, utilizando los motores de búsqueda PubMed, ScienceDirect y Google Scholar con los términos de búsqueda libre en el idioma inglés: Free hand in scoliosis, Free hand pedicle screw placement, Pedicle screw thoracic y complication of free hand pedicle screw placement. A partir de los resultados se escogieron 22 artículos que se ajustaran a la explicación de la técnica, complicaciones y curvas de aprendizaje de esta práctica.

\section{Técnica de colocación de tornillos pediculares}

Lenke y cols. describieron la experiencia y la técnica de fijación de asideros transpediculares torácicos con el método de manos libres, reportando su experiencia de 10 años. ${ }^{4}$ Desde esa publicación, varios autores han hecho modificaciones y anexos a la modalidad original descrita por Lenke. ${ }^{4}$ Esto lleva a tener diferentes criterios, desde el punto de entrada, disección anatómica, hasta el tipo de instrumental para la perforación del pedículo (Figura 1). ${ }^{5}$

Con base en la literatura reportada, se describirá mediante pasos la instalación de tornillería transpedicular con el sistema de manos libres de esta manera:

1. Con el paciente en decúbito prono, se realiza incisión en la línea media, abordaje donde se preservan los elementos del arco posterior. Se expone la lámina vertebral, apófisis transversa y faceta articular superior e inferior de cada nivel que se quiere instrumentar.

2. Facetectomía: se osteotomizan los tres a cinco milímetros de la faceta inferior de cada vértebra. Conjuntamente se retira la cápsula articular de la faceta superior. Sin embargo, en las facetas de los niveles extremos de instrumentación se pre- serva la cápsula articular con el objetivo de no dañar la estabilidad ligamentaria.

3. Localización del punto de entrada: inicialmente, ubicarse de caudal a cefálico en la vértebra T12, lo cual va a permitir identificar un punto de ingreso distinto que será cada vez más hacia la media y cefálico a medida que avanza de nivel hasta llegar al ápice (T8-T7). Proximal a eso, el punto de acceso tiende a moverse más lateral y caudal hasta llegar a vértebra $\mathrm{T}^{4}$ (Figura 2). Varios autores han propuesto simplificar el punto de entrada único en columna no escoliótica, el cual se localiza tres milímetros caudal a la confluencia del borde superior de apófisis transversa y borde lateral de la faceta articular, al tener en cuenta la inclinación de 30 grados medial en T1 y T2, cambiando a 20 grados medial desde T3 a T12; al mismo tiempo conservando la alineación sagital, ortogonal de cada vértebra $^{6}$ (Figura 3). Otro punto de ingreso, basado en el planeamiento de corte axial por tomografía computarizada de la faceta articular superior de la vértebra a instrumentar, teniendo vértice de 90 grados en relación con la superficie articular, que corresponde al trayecto central del pedículo en $90 \%$ de las vértebras torácicas ${ }^{7}$ (Figura 4).

4. Canalización del pedículo: una vez ubicado el punto de introducción, se coloca lezna curva con una angulación de lateral a medial de 15-20 grados para los niveles T12 a T3 y 20 a 30 grados en los niveles T2-T1. Se debe tener en cuenta el plano sagital a fin de determinar la angulación caudal o cefálica de cada vértebra. Dirigir la lezna 15-20 milímetros con la concavidad a lateral buscando evitar lesión de pared en la media

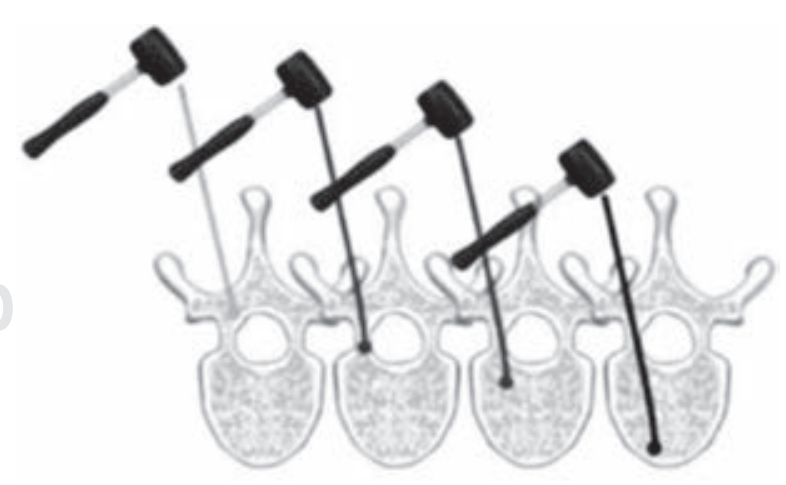

Figura 1: llustración de técnica representando la forma que usan los autores. Si el instrumento punta-bola encuentra algo de resistencia puede usar martillos con golpes suaves. ${ }^{5}$ 

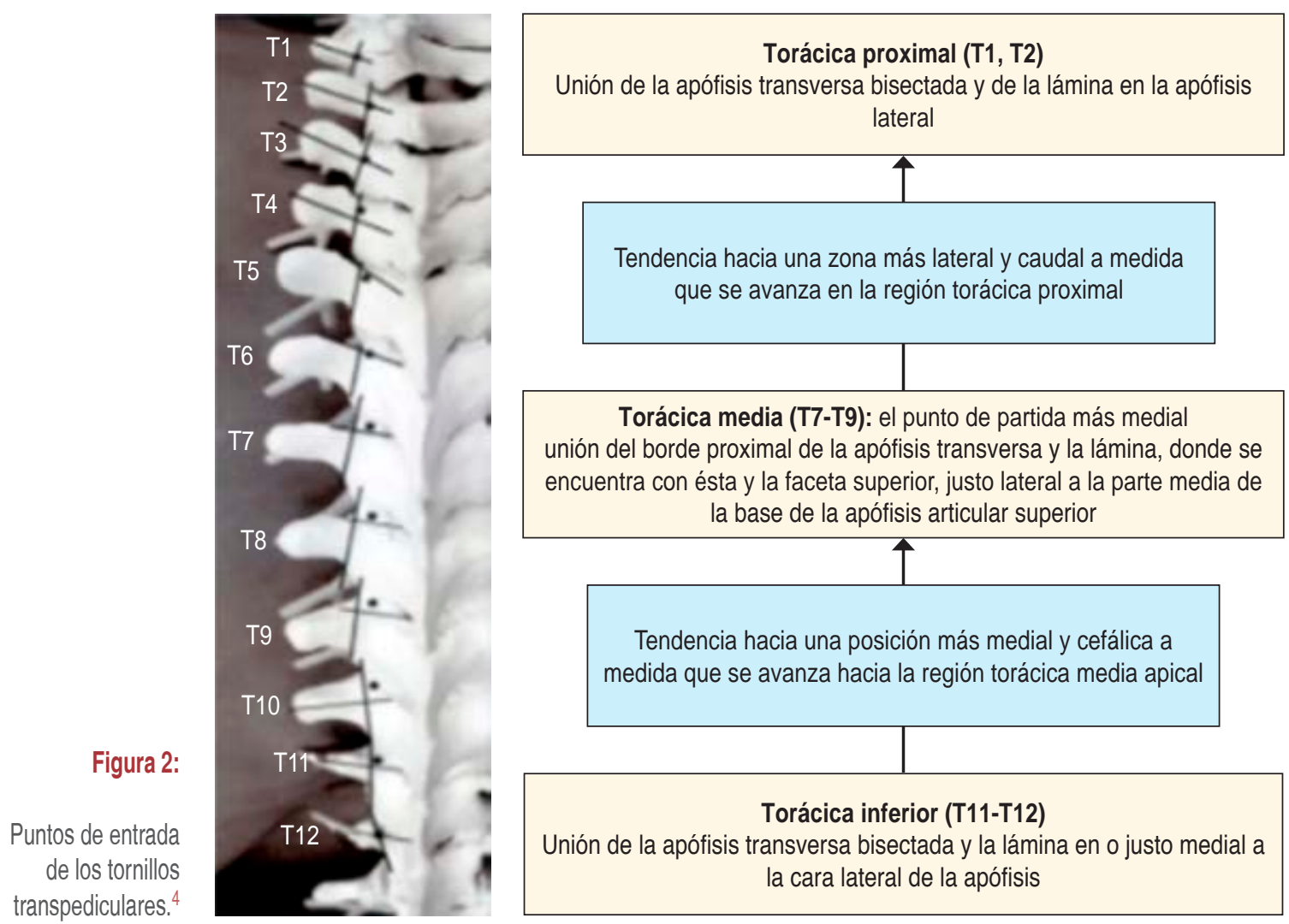

pedicular, retirar la lezna y girarla 180 grados cambiando a medial la concavidad, permitiendo dirigirse al cuerpo vertebral con movimientos oscilatorios hasta la profundidad previamente planificada que varía de 30 a $45 \mathrm{~mm}$ (Figura 5).

5. Corroborar la integridad por medio de palpación del fondo y las cuatro paredes, haciendo énfasis en la pared hacia la media. Al mismo tiempo, se mide la longitud del pedículo con el palpador y pinza hemostática en el punto de entrada.

6. Roscado: introducir un machuelo con un diámetro un milímetro menor al del tornillo deseado, para facilitar el trayecto del mismo, lo que facilitará el anclaje y posicionamiento del tornillo final.

7. Segunda palpación: se corrobora de nuevo la integridad de las paredes y el fondo pedicular, a modo de evitar rupturas del pedículo.

8. Colocación del tornillo: se sitúa este elemento lentamente en la misma dirección que proporciona el palpador, esto con el fin de evitar vía falsa. Realizar la instalación de esta manera permite una expansión hasta de $200 \%$ del diámetro del pedículo pediátrico, facilitando introducir tornillería con mayor diámetro al del pedículo.4,8

\section{Clasificación de colocación de tornillos transpediculares}

La fijación del tornillo transpedicular puede generar lesiones neurológicas y/o vasculares, si se

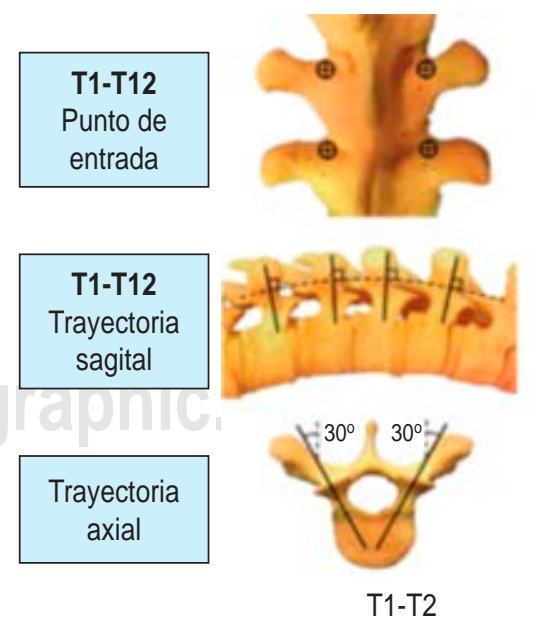

Proceso articular de $3 \mathrm{~mm}$ caudal a la unión de la apófisis transversa superior

Ortogonal a la curvatura sagital de la espina dorsal

Figura 3: Colocación de tornillo transpedicular utilizando punto de entrada único y trayectoria sagital con ángulos axiales apropiados. ${ }^{6}$ 


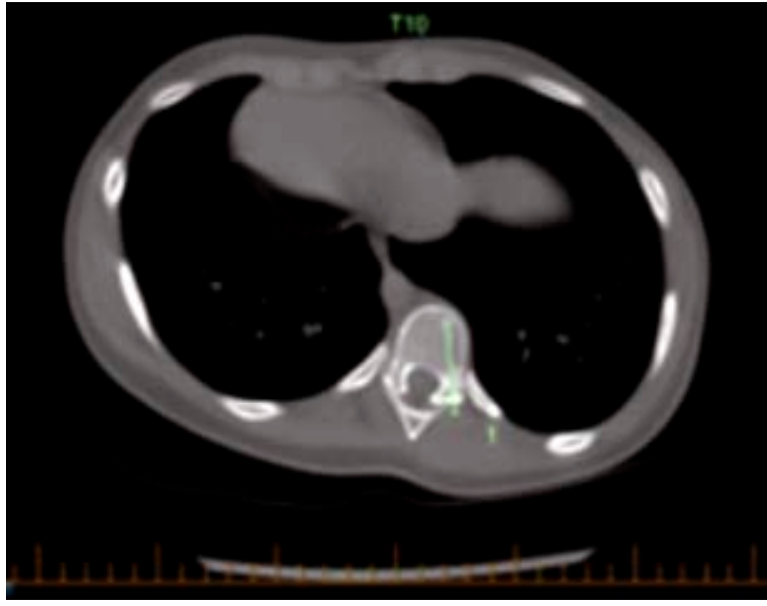

Figura 4: Técnica de medición; una línea perpendicular de la superficie del proceso facetario articular superior en tomografía axial computarizada. ${ }^{7}$

coloca de manera incorrecta. Como consecuencia de no haber un criterio estandarizado, debido a la variación de tamaño de los pedículos, además de variantes anatómicas y patológicas, se crearon estudios que proponen medidas de cortes axiales a nivel pedicular, determinando la zona de seguridad que puede ser usada en columnas con deformidad o sin ésta ${ }^{9}$ (Figura 6).
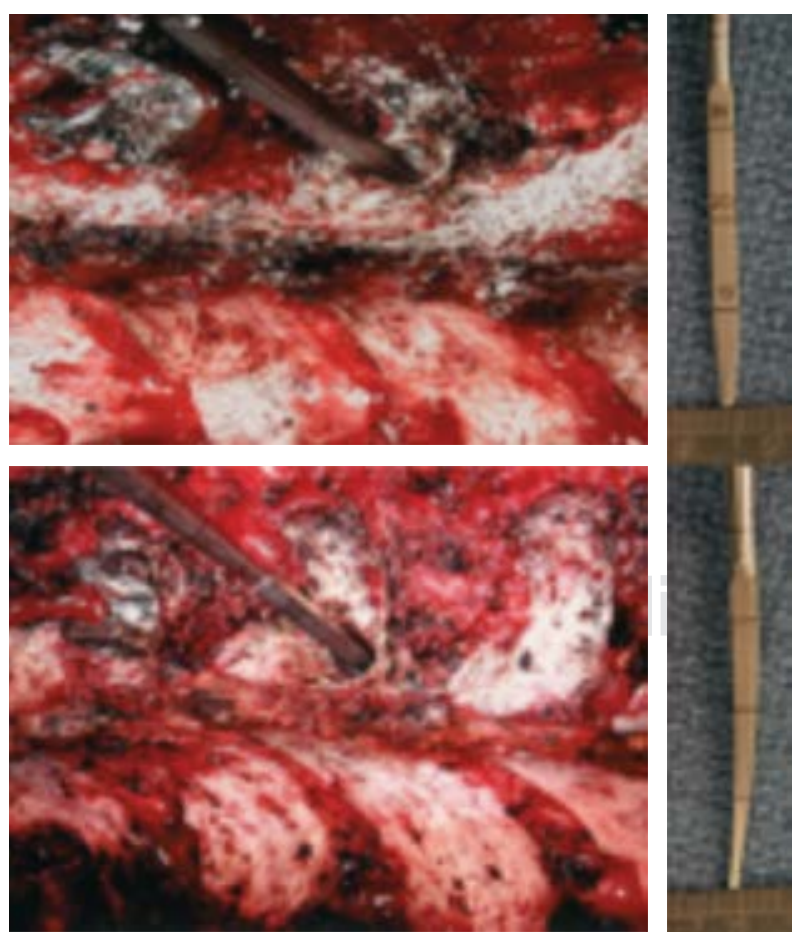

Las zonas de seguridad se dividen por grados, siendo el cero una colocación exacta en el pedículo y aumenta su valor con respecto a la imprecisión del posicionamiento del elemento roscado. En términos generales, se puede consensuar en la mayoría de la literatura que una zona segura corresponde a un grado $0-1$ como se determina. ${ }^{10}$

- Grado 0: totalmente contenido en el pedículo.

- Grado 1: penetración hasta $2 \mathrm{~mm}$ medial y $4 \mathrm{~mm}$ lateral.

- Grado 2: penetración de 2.1-4 mm medial y 6 $\mathrm{mm}$ lateral.

- Grado 3: penetración de 4.1-6 mm medial y más de $6 \mathrm{~mm}$ lateral.

- Grado 4: mayor a $6 \mathrm{~mm}$.

\section{Uso de la técnica en columnas escolióticas y no escolióticas}

La evolución de la estrategia de manos libres, en conjunto con la cirugía de columna, crearon la posibilidad de disminuir la necesidad de imágenes intraoperatorias. Las tres patologías en las que se da uso al tornillo transpedicular son; etiología no escoliótica, escoliosis idiopática del adolescente y escoliosis de origen neuromuscular.
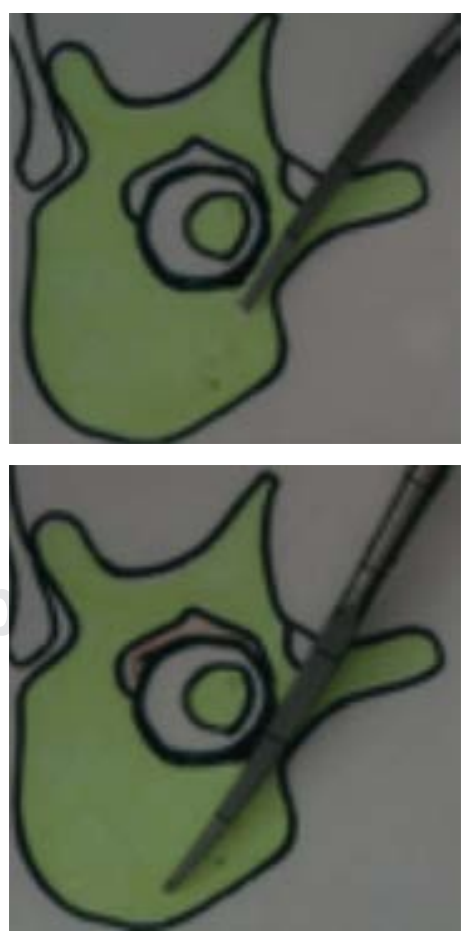

Figura 5:

Prueba de colocación de la lezna con una profundidad de 20 milímetros para disminuir la probabilidad de riesgo de perforación del pedículo, posteriormente retirar la lezna y redirigirla medialmente. ${ }^{4}$ 
Figura 6:

Tipos de clasificación de la colocación de tornillos

transpediculares.

A) Tornillo completamente dentro del pedículo.

B) Tornillos con

acercamiento a pared medial pedicular, dentro del pedículo. C) Tornillo

transpedicular con

penetración de $2 \mathrm{~mm}$ de

la pared medial.

D) Tornillo transpedicular

torácico dentro de la articulación costovertebral

(in-out-in). ${ }^{9}$
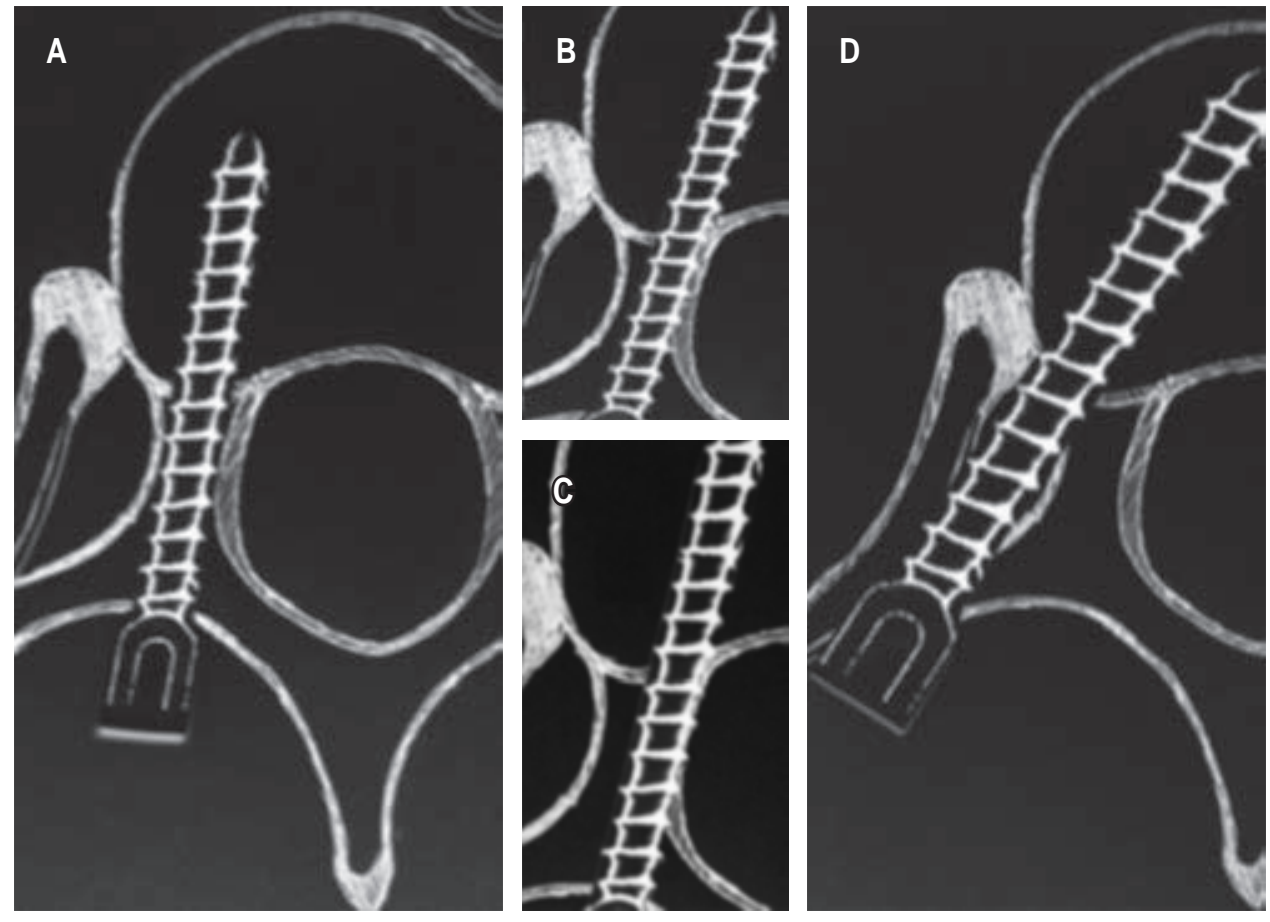

La necesidad de disminuir al mínimo la exposición a la radiación en cirugías de columnas no escolióticas llevó a que se perfeccione el conocimiento de la anatomía vertebral y sus relaciones neurovasculares. Esto con el fin de tener una seguridad equiparable a técnicas donde se usen imágenes intraoperatorias. ${ }^{11}$

En el manejo de columnas con algún tipo de deformidad, ${ }^{12,13}$ demostraron la validación de fijación de tornillería transpedicular con el método de manos libres en escoliosis idiopática del adolescente, con curvas estructuradas severas con más de 80 grados de curva principal. Se presentó $65.2 \%$ de colocación grado 0 , con reporte de rupturas pediculares mediales de $13.1 \%$ y laterales de $21.6 \%$, que corresponden al $90.7 \%$ de instalación en la zona segura (grado 1).

En cuanto a las escoliosis neuromusculares, un estudio realizó un análisis donde se reportó la experiencia en seguridad de introducción de tornillos transpediculares a manos libres en deformidades severas. De estos elementos, se reporta que $73 \%$ se colocaron con precisión dentro del pedículo (grado 0) y 93.3\% corresponden a la zona de seguridad (grado 1). ${ }^{10}$

Lo anterior evidencia que el uso de estas piezas transpediculares tienen suficiente soporte literario científico para la instrumentación de columnas de diferentes patologías con una seguridad equiparable, resultando en su mayoría de colocación entre grado 0 y 1 sin complicaciones neurovasculares.

\section{Curva de aprendizaje}

Los años de experiencia de los cirujanos juegan un papel fundamental en la técnica de manos libres, como lo demuestra el estudio realizado por Samdani y compañía, donde se compararon tres conjuntos de cirujanos según sus años de experiencia. Los equipos se dividieron de la siguiente manera, de acuerdo a su pericia: grupo 1 (menos de dos años), grupo 2 (de 2 a 5 años), grupo 3 (más de 5 años). En el estudio se observó una diferencia significativa en cuanto a lesión medial del pedículo de $7.4 \%$ en el grupo 1 , versus $3.5 \%$ en grupo $3 .{ }^{14}$ Así mismo, Chen y colegas compararon la colocación de tornillería por un cirujano jefe versus un cirujano aprendiz, donde se contrastaron 154 de estos elementos y se observaron más rupturas mediales al inicio del entrenamiento del aprendiz, sin embargo, posterior a la fijación de 60 tornillos transpediculares se observaron resultados similares a los del cirujano jefe. ${ }^{15}$

A fin de valorar la curva de aprendizaje se realizó un estudio con columnas cadavéricas, las cuales fueron instrumentadas por tres cirujanos en entrenamiento. Dentro de este estudio se usaron 15 columnas 
vertebrales y se instrumentaron 297 pedículos, en los cuales se observó $29 \%$ de rupturas del pedículo en un grado. Se reportó $3.1 \%$ de rupturas mediales críticas de más de 4 milímetros. Esta observación se encontró en los tres primeros especímenes de cada cirujano y disminuyó a partir del cuarto espécimen en adelante. Se determinó que se tiene una condición de confianza y aprendizaje posterior a la colocación de 80 tornillos pediculares. ${ }^{16}$

Para los cirujanos con poca experiencia en el uso de estos elementos transpediculares se recomienda el uso de herramientas de ayuda en la instalación de los mismos. En una revisión retrospectiva de instrumentaciones en escoliosis idiopática del adolescente, se observó que, a medida que progresa la curva de aprendizaje, disminuyen los tiempos quirúrgicos y hay mejores desenlaces en los pacientes. Por lo tanto, los autores sugieren a los cirujanos sin experiencia iniciar con técnicas de apoyo como fluoroscopia y laminotomía para palpación interna de los pedículos. ${ }^{17}$

En la colocación de tornillería transpedicular, para reconocer lesión medular se propone el uso de neuromonitoreo y otras herramientas de ayuda, según la experticia del cirujano. En contraste, en un reporte de 120 pacientes con 1,004 sujetadores pediculares colocados por un cirujano jefe, sin uso de neuromonitorización, ni fluoroscopia transquirúrgica, no se tuvieron casos de lesión neurológica, visceral o vascular; revelando la seguridad del método en manos expertas. ${ }^{18}$

A medida que el cirujano de columna va aumentando su experticia y seguridad con este tipo de técnica, la incidencia de rupturas de las paredes pediculares va disminuyendo. ${ }^{19}$ Se realizó un estudio retrospectivo donde se operaron 10 pacientes por grupo, siendo el grupo A los primeros 10 pacientes, el $B$ los siguientes 10 y el grupo $C$ los últimos 10 pacientes. A medida que los cirujanos iban operando, por conjunto se demostró una disminución notable de infracciones generales (grupo A, 15.5\%; el B, 10.6\%; grupo $C, 8.7 \% ; p<0.05$ ) y rupturas mediales (grupo A, 9.4\%; grupo B, 5.8\%; y C, 3.8\%; $p<0.05$ ). De acuerdo con lo anterior, en la inserción de tornillos transpediculares por la modalidad de manos libres se necesita una curva de aprendizaje corta y reproducible en la mayoría de los centros de entrenamiento.

\section{Complicaciones}

El uso generalizado de la técnica manos libres puede presentar una incertidumbre en los cirujanos de columna, donde se valora la exposición a radiación y tiempos prolongados versus riesgos neurovasculares. Con el fin de disminuir los riesgos, diversos autores proponen alternativas combinadas y variantes de apoyo a la técnica manos libres. ${ }^{20}$ En ellos se pueden encontrar microlaminectomías para la palpación de la pared medial pedicular y otras ayudas más. Esto se realizó con el propósito de disminuir las complicaciones, como ruptura de la pared pedicular, lesión de duramadre, lesiones tardías de aorta entre otras.

La aplicación del procedimiento de manos libres ha demostrado su seguridad y exactitud por medio de una revisión sistemática ${ }^{21}$ donde se incorporaron 21 estudios que incluyeron 1,666 pacientes con escoliosis idiopática del adolescente, dando un total de 4,570 tornillos transpediculares, en torno a los cuales se reportó una colocación inadecuada de 11 a 15.7\%, de este porcentaje, se observaron principalmente estragos como
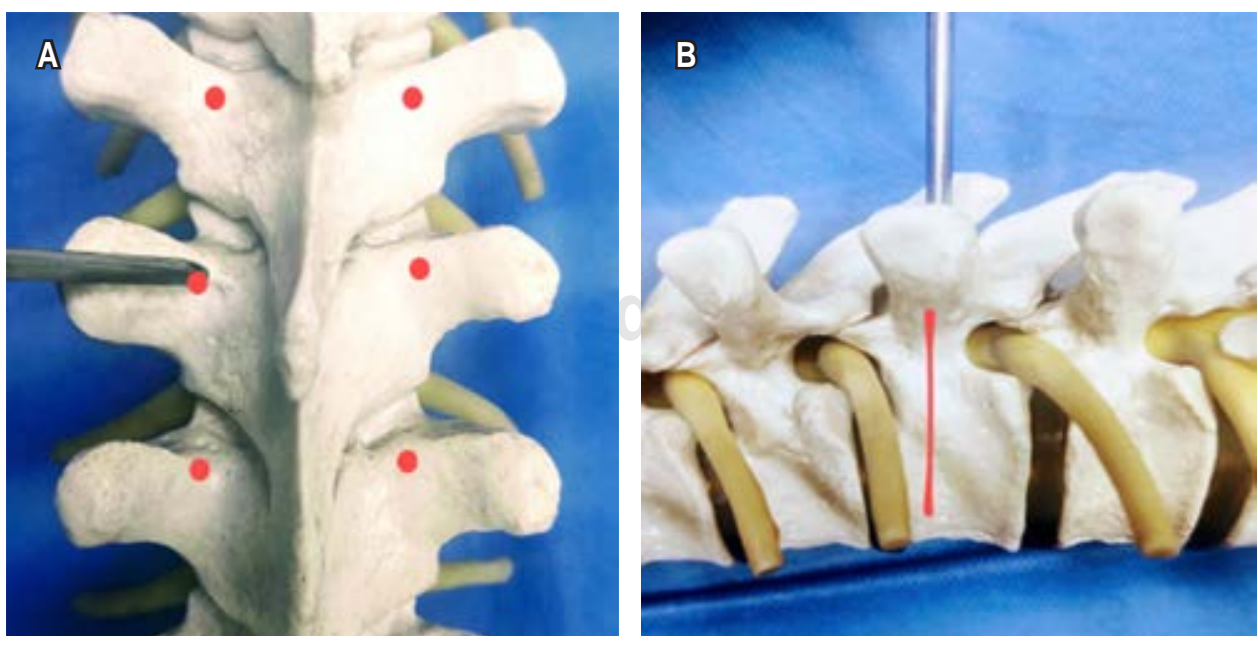

Figura 7:

A) Muestra puntos de entrada a $2 \mathrm{~mm}$ de la confluencia facetatransversa. B) Vista sagital que muestra trayecto pedicular desde el punto de ingreso. 
ruptura de pared lateral del pedículo y que las lesiones neurovasculares o pulmonares son raras y de baja incidencia. De igual manera, un estudio retrospectivo donde se reportó la experiencia de 12 años con 486 pacientes con escoliosis idiopática del adolescente, demostró que sólo $0.6 \%$ requirieron reintervención quirúrgica por no estar en zona segura (grado 1), en los cuales transquirúrgicamente se corroboró ruptura del saco dural sin lesión neurológica, y ningún paciente presentó lesión vascular o visceral. ${ }^{22}$ Como resultado, recomiendan el uso de técnica manos libres a cirujanos de columna vertebral con adecuado entrenamiento en la misma.

\section{Conclusiones}

Basado en la literatura establecida, se sugiere el uso generalizado del procedimiento manos libres para los cirujanos de columna. Es importante recalcar que los médicos en adiestramiento deberían iniciar las cirugías supervisadas por cirujanos en jefe y apoyándose al inicio en técnicas imagenológicas u otras alternativas, con el fin de tener una curva de aprendizaje sin complicaciones.

Al tener en cuenta que existen diversos puntos de ingreso descritos en la literatura con una mínima variación entre los niveles a instrumentar, se recomienda identificar un punto promedio de entrada, el cual se localiza dos milímetros inferior y medial a la confluencia del borde superior de la apófisis transversa y borde lateral de faceta articular superior de la vértebra a instrumentar, teniendo en cuenta la alineación ortogonal de cada vértebra para el plano sagital (Figura 7).

\section{Referencias}

1. Boucher HH. A method of spinal fusion. J Bone Joint Surg Br. 1959; 41-B (2): 248-259.

2. Denis F. The three column spine and its significance in the classification of acute thoracolumbar spinal injuries. Spine (Phila Pa 1976). 1983; 8 (8): 817-831.

3. Falavigna A, Ramos MB, lutaka AS, Menezes CM, Emmerich $\mathrm{J}$, Taboada N, et al. Knowledge and attitude regarding radiation exposure among spine surgeons in Latin America. World Neurosurg. 2018; 112: e823-e829.

4. Kim YJ, Lenke LG, Bridwell KH, Cho YS, Riew KD. Free hand pedicle screw placement in the thoracic spine: is it safe? Spine (Phila Pa 1976). 2004; 29 (3): 333-342; discussion 342.

5. Watanabe K, Matsumoto M, Tsuji T, Ishii K, Takaishi H, Nakamura M, et al. Ball tip technique for thoracic pedicle screw placement in patients with adolescent idiopathic scoliosis. J Neurosurg Spine. 2010; 13 (2): 246-252.

6. Fennell VS, Palejwala S, Skoch J, Stidd DA, BaajAA. Freehand thoracic pedicle screw technique using a uniform entry point and sagittal trajectory for all levels: preliminary clinical experience. J Neurosurg Spine. 2014; 21 (5): 778-784.
7. Stevanovic O, Hermanson AR, Anderson JT. Is the superior articular process a reliable landmark for determining the transverse plane angulation of thoracic pedicles in patients with adolescent idiopathic scoliosis? Spine Deform. 2017; 5 (1):52-55.

8. Kim YJ, Lenke LG. Thoracic pedicle screw placement: freehand technique. Neurol India. 2005; 53 (4): 512-519.

9. Upendra BN, Meena D, Chowdhury B, Ahmad A, Jayaswal A. Outcome-based classification for assessment of thoracic pedicular screw placement. Spine (Phila Pa 1976). 2008; 33 (4): 384-390.

10. Modi HN, Suh SW, Fernandez H, Yang JH, Song HR. Accuracy and safety of pedicle screw placement in neuromuscular scoliosis with free-hand technique. Eur Spine J. 2008; 17 (12): 1686-1696.

11. Ahmadzadeh Heshmati A, Ilka S. Accuracy assessment of freehand pedicular screw placement. Arch Bone Jt Surg. 2020; 8 (4): 519-523.

12. Gatam L, Luthfi APWY, Fachrisal, Phedy, Gatam AR, Djaja YP. A posterior-only approach for treatment of severe adolescent idiopathic scoliosis with pedicle screw fixation: A case series. Int J Surg Case Rep. 2020; 77: 39-44.

13. Modi HN, Suh SW, Hong JY, Yang JH. Accuracy of thoracic pedicle screw using ideal pedicle entry point in severe scoliosis. Clin Orthop Relat Res. 2010; 468 (7): 1830-1837.

14. Samdani AF, Ranade A, Sciubba DM, Cahill PJ, Antonacci $\mathrm{MD}$, Clements $\mathrm{DH}$, et al. Accuracy of free-hand placement of thoracic pedicle screws in adolescent idiopathic scoliosis: how much of a difference does surgeon experience make? Eur Spine J. 2010; 19 (1): 91-95.

15. Gang C, Haibo L, Fancai L, Weishan C, Qixin C. Learning curve of thoracic pedicle screw placement using the freehand technique in scoliosis: how many screws needed for an apprentice? Eur Spine J. 2012; 21 (6): 1151-1156.

16. Bergeson RK, Schwend RM, DeLucia T, Silva SR, Smith JE, Avilucea FR. How accurately do novice surgeons place thoracic pedicle screws with the free hand technique? Spine (Phila $\mathrm{Pa}$ 1976). 2008; 33 (15): E501-E507.

17. Lonner BS, Auerbach JD, Estreicher MB, Kean KE. Thoracic pedicle screw instrumentation: the learning curve and evolution in technique in the treatment of adolescent idiopathic scoliosis. Spine (Phila Pa 1976). 2009; 34 (20): 2158-2164.

18. Crostelli M, Mazza O, Mariani M. Free-hand pedicle screws insertion technique in the treatment of 120 consecutive scoliosis cases operated without use of intraoperative neurophysiological monitoring. Eur Spine J. 2012; 21 Suppl 1(Suppl 1): S43-S49.

19. Samdani AF, Ranade A, Saldanha V, Yondorf MZ. Learning curve for placement of thoracic pedicle screws in the deformed spine. Neurosurgery. 2010; 66 (2): 290-294; discussion 294-295.

20. Di Silvestre M, Parisini P, Lolli F, Bakaloudis G. Complications of thoracic pedicle screws in scoliosis treatment. Spine (Phila Pa 1976). 2007; 32 (15): 1655-1661.

21. Hicks JM, Singla A, Shen FH, Arlet V. Complications of pedicle screw fixation in scoliosis surgery: a systematic review. Spine (Phila Pa 1976). 2010; 35 (11): E465-E470.

22. Dede O, Ward WT, Bosch P, Bowles AJ, Roach JW. Using the freehand pedicle screw placement technique in adolescent idiopathic scoliosis surgery: what is the incidence of neurological symptoms secondary to misplaced screws? Spine (Phila Pa 1976). 2014; 39 (4): 286-290.

\section{Conflicto de intereses}

Los autores no presentan ningún conflicto de intereses. 